\title{
Multiple-choice test of energy and momentum concepts
}

\author{
Chandralekha Singh and David Rosengrant \\ Department of Physics and Astronomy, \\ University of Pittsburgh, Pittsburgh, PA 15260
}

\begin{abstract}
We investigate student understanding of energy and momentum concepts at the level of introductory physics by designing and administering a 25-item multiple choice test and conducting individual interviews. We find that most students have difficulty in qualitatively interpreting basic principles related to energy and momentum and in applying them in physical situations.
\end{abstract}




\section{INTRODUCTION}

Research-based multiple-choice tests can be powerful probes of student understanding. They are easy and economical to administer and to grade, have objective scoring, and are amenable to statistical analysis that can be used to compare student populations or instructional methods. A major drawback is that the thought processes are not revealed completely by the answers alone. However, when combined with student interviews, welldesigned tests are powerful tools for educational assessment. Conceptual multiple-choice tests have already been designed to assess student understanding of force, kinematics graphs,

heat, circuits, and electricity and magnetism. ${ }^{1}$ They suggest that students' knowledge of physics often is fragmented and context-dependent, and that students have many common difficulties.

Energy and momentum are two of the most fundamental concepts in physics..$^{2}$ In the lower-level physics courses, they are typically introduced after Newton's laws. We have designed and administered a research-based 25-item multiple-choice test and conducted individual interviews to explore student understanding of energy and momentum concepts. $\underline{3}$ We are interested in understanding the difficulties students have in interpreting these concepts and in correctly identifying and applying them in different physical situations. We also wish to know the extent to which the difficulties are universal, and if there is a correlation with student preparation (for example, calculus or algebra background). The identification of student difficulties can help in designing instructional tools that address them. Part of the rationale for combining energy and momentum concepts is to investigate the extent to which students can identify the relevant concept in a particular situation.

\section{TEST DESIGN}

During the test design, we paid particular attention to the important issues of reliability and validity $\stackrel{4}{*}$ Reliability refers to the relative degree of consistency in scores between testing if the test procedures are repeated for an individual or group. Validity refers to the appropriateness of the test score interpretation. The test design began with the development of a test blueprint that provided a framework for planning decisions about the desired test attributes. We tabulated the scope and extent of the content covered and the level of cognitive 
complexity desired. The specificity of the test blueprint was useful for creating questions. The energy concepts included the work-energy theorem, conservation of mechanical energy, and work done by gravitational and frictional forces. The momentum concepts included the definition of momentum, impulse-momentum theorem (impulse was defined explicitly in the test), and conservation of momentum with examples from elastic and inelastic collisions. We also planned to evaluate student understanding of the concept of "system" in various contexts. We also planned to include one question that explicitly required the application of both energy and momentum concepts. Energy and momentum questions pertaining to simple harmonic motion, explicit mention of conservative and non-conservative forces and center-of-mass reference frame were deliberately excluded, because we wanted the test content to be commensurate with the curriculum in most calculus- and algebra-based courses for science and engineering majors. We classified the cognitive complexity using a simplified version of Bloom's taxonomy: $:^{\frac{5}{-}}$ specification of knowledge, interpretation of knowledge and drawing inferences, and applying knowledge to different situations. Then, we outlined a description of conditions/contexts within which the various concepts would be tested and a criteria for good performance in each case . The tables of content and cognitive complexity along with the criteria for good performance were shown to five physics faculty members at the University of Pittsburgh for review. Modifications were made to the weights assigned to various concepts and to the performance criteria based upon the feedback from the faculty about their appropriateness. The performance criteria was used to convert the description of conditions/contexts within which the concepts would be tested to make approximately 50 free-response questions. These questions required students to provide their reasoning for their responses.

The free-response questions were administered (in groups of 10 or 20) to students in the calculus- and algebra-based courses at the University of Pittsburgh in the Fall of 1998. Often, some students in a class were given one set of questions and others were given another set in order to sample student responses on most of the questions. We also tape-recorded interviews with 10 introductory student volunteers using the think-aloud protocol. $\underline{6}$ Forty multiplechoice items (questions) were then designed using the most frequent student responses for the free-response questions and interviews as a guide for making the alternative distractor choices. Four alternative choices have typically been found to be optimal ${ }^{4}$ and we chose the four distractors to conform to the common difficulties to increase the discriminating 
properties of the items. Ten physics faculty members and postdocs were asked to review the multiple-choice items and comment on their appropriateness and relevance for introductory physics and to detect ambiguity in item wording. An item review form was developed to aid the faculty in reviewing the items. The faculty also classified each item on a scale from very appropriate to least appropriate. Further modifications were made based upon their recommendation. Then, a multiple-choice test was assembled using 28 items which closely matched the initial table delineating the scope of the content and cognitive complexity. The same faculty members who reviewed the items were shown the test and some modifications were made.

The test was administered as a 50-minute post-test (after instruction) to several hundred students in calculus- and algebra-based courses at the University of Pittsburgh in Spring 1999. Seven student volunteers who had taken the test were interviewed using the thinkaloud protocol. Item analysis of student responses was performed to judge the quality of each

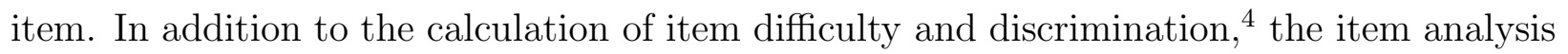
included creating a table to count the number of students selecting each distractor in the upper and lower quartiles. This analysis was very useful to determine whether individual items and distractors functioned as expected. Based upon the item analysis and interviews, the test items were modified further. The number of items in the test was decreased to 25 . Including the spring and fall 1999, 2000 and 2001 the test has been administered to more than 3000 students from approximately 30 calculus- and algebra-based courses in different colleges and universities. Some classes administered the test both as a pre-/post-test to assess students' conceptions of energy and momentum before instruction, the effectiveness of the instruction, and the effect of pre-test on post-test. Every semester an item analysis was performed after its administration, a few students were interviewed, and some items were slightly modified. We have conducted a total of 34 one-hour interviews during the test development process. In Fall 2000, the test was administered to graduate students enrolled in a teaching methods class. Their average score was greater than $80 \%$ and the reliability coefficient $\underline{4}^{4} \alpha$ (which is a measure of the internal consistency of the test ${ }^{4}$ ) was greater than 0.8 . 


\section{RESULTS}

The final version of the test is included in Appendix A. The test was administered before the instruction on energy and momentum concepts (pre-test) only in some classes. The average pre-test scores typically ranged from $24 \%-36 \%$ depending upon the class type, for example, calculus versus algebra (higher for calculus), and whether the test was administered before or after any instruction on linear kinematics/dynamics (some questions can be answered without explicitly invoking energy or momentum concepts).

We first discuss the post-test results of the version administered to 1356 students in Fall 2000. The reliability coefficient $\alpha$ for the calculus-based classes was slightly more than 0.75 (1170 students), while that for the algebra-based classes was 0.68 (186 students). Figures 1 and 2 show the post-test statistics for the calculus-based courses (1170 students) including the honors (two) and active-engagement classes (one). The average post-test score was $49 \%$ (standard deviation $\sigma=18 \%$ ). Figure 1 shows a plot of the item difficulty (the fraction of students who got the item correct), which ranged from 0.2 to 0.8 . This range is consistent with our goal because we want to explore student performance on items with a variety of cognitive complexity. Figure 2 shows a plot of the point biserial discrimination (PBD) 4 for individual items which measures the ability of an item to distinguish between high (those who performed well on the test overall) and low performing students. The PBD ranged from 0.21 to 0.48 with only one item with PBD less than 0.28 , which is good by the standards of achievement test design. $\underline{4}$ [xx it looks like you left out some references to Table I and II which were in the hard copy from Redish $\mathrm{xx}$ ] The average score on the items focusing mainly on the energy concepts (14 total) was $46 \%(\sigma=20 \%)$, while that on items focusing mainly on momentum concepts (10 items) was $55 \%(\sigma=22 \%)$. There was a reasonable correlation between the performance on the energy questions and momentum questions (Pearson correlation ${ }^{4}$ 0.54). We performed a factor analysis (principal component method) ${ }^{7}$ which identified six factors with a variance greater than $4 \%$. However, the largest of these factors accounted for less than $15 \%$ of the variance. This variance is very small for the first factor so none of the factors are meaningful. Although the analysis of variance (ANOVA) 4 shows statistically significant differences between several calculus-based classes at the level of $\alpha=0.05$, the effect size $d$ is small $(d<0.35)$ for all pairs except those involving an honors class or an active-engagement class. $\stackrel{-}{-}$ Also, ANOVA shows a statistically significant 
difference between the calculus-based (excluding the honors and active-engagement classes) and algebra-based classes in terms of the total average score, but again the effect size is small. ANOVA on individual items shows that the differences between the calculus- and algebra-based classes are not statistically significant for 12 of the 25 test items.

\section{A. Pre/Post Instruction Analysis}

To assess the effect of instruction, we now discuss results (unmatched) from three calculusbased classes for which both pre-/post-tests were administered. A total of 352 and 336 students from the three classes took the pre-test and post-test in Fall 2001 and the average scores were $33.6 \%$ and $51.7 \%$ respectively. The average post-test score was more than $50 \%$ on 15 out of the 25 items. Table I shows the average percent correct on the pre-/post-test and the normalized gain $g=\left(s_{f}-s_{i}\right) /\left(100-s_{i}\right)$, where $s_{i}$ and $s_{f}$ are the pre-/post-test scores in percent $)^{\frac{9}{}}$ on each test item separately for students whose total test scores were in the upper 25\% (group H), lower 25\% (group L), and all students (A). The average pre-test scores of students in the upper and lower quartiles were $49.8 \%$ and $19.2 \%$ and the average post-test scores in these two groups were $70.6 \%$ and $32.2 \%$ respectively. Thus, students in the upper quartile gained significantly more than those in the lower quartile with an average gain of 0.45 for group H, 0.16 for group L, and 0.29 for all students A. The upper and lower quartiles were selected based the average scores on the pre and post tests. Table II shows the distribution of alternative choices for the pre-/post-tests. It shows the types of preconceptions students had before instruction on the energy and momentum concepts and how the conceptions changed after the instruction. Comparison of the pre-/post-tests in Table II shows that instruction improved student conceptions in some cases, but not in others.

The test results reveal that students lack a coherent understanding of energy and momentum concepts and have difficulty applying them to different physical situations. They often focused on the surface features and were distracted by irrelevant details. ${ }^{10}$ During the interview, some students suggested that the lack of numerical values for the parameters prevented them from checking if they are on the right track. Many students did not realize that work and energy are scalar quantities and momentum is a vector quantity. Questions involving work-energy or impulse-momentum theorems were typically perceived to be more 
difficult than those involving their special cases: the conservation of mechanical energy and momentum respectively. However, students had great difficulty in using the conservation principles appropriately in many situations. In the following, we discuss some insightful features of student responses by loosely clustering items based upon the relevant physics concepts. Although the same item can be categorized in different ways, we choose to discuss them within a particular cluster.

Questions related to energy concepts. Items 2, 4, 15, and 22 can all be answered based upon the conservation of mechanical energy, because there is no work done by nonconservative forces. (The normal force is present in the "slide" related items (2) and (15), but it is perpendicular to the direction of motion of the object, so it does no work on it.) Students performed very differently on these items and did not perform any better on items (4) and (22) which involved only the gravitational force compared to items 2 and 15 which had a normal force present. Interviews show that in many physical situations students knew "what" but did not know "why." For example, in response to item 4, several interviewed students said that the balls thrown from a cliff will reach the ground at the same speed, regardless of the angle of projection, but they could not justify their answers based either upon energy or kinematic considerations. Item 15 elicits strong belief that "the heavier person has a larger speed at the bottom of the slide because the larger weight causes a greater acceleration," a belief which did not improve much after instruction. An interviewed student noted: I remember my dad used to slide down faster because his mass carried him down more. Another student who chose (c) used correct reasoning except in the last step where he did not realize that the mass will not affect the speed: . . another way to see it is that your potential energy is larger than your niece's ... your kinetic energy will be larger at the bottom ... so your speed is larger. If it were a numerical problem, there is a chance that this student would have solved it correctly, perhaps without consciously noting that the mass drops out. For item 22, interviews indicate that students who viewed this as a projectile motion problem were very likely to get it wrong. A student who chose (a) explained: ball (1) gets there fastest so it will not lose its speed as much ... and for the same reason ball (2) will be faster than ball (3). Another student had something similar to say: the longer the path ... more chance gravity has to slow the ball down. A student who chose (c) explained: ball thrown vertically will slow down most because all its motion is in the $y$ direction ... the more is the component in the $x$ direction ... the more it remains unchanged. 
The student is probably confusing the time it takes to reach a height with the speed at that height, and he is overlooking the fact that all the three balls are at the same height. Another student who chose (c) explained: ... smaller angle [with horizontal] will reduce downward acceleration making the speed larger. Incidentally, in item 22 , in the post-test, only $10 \%$ from group L chose (e) indicating that the speed of a ball at a given height depends on the mass, but $60 \%$ indicated in item 15 that the speed of an object sliding down the frictionless slide depends upon mass. This context dependent response suggests that students' knowledge is fragmented and their recall is triggered by surface features rather than the underlying physics concepts.

Items $1,6,8$, and 20 probe understanding of work done by the gravitational force. Response to item 1 shows the pervasive belief that the work done by the gravitational force is path-dependent. Part of the problem is the difference between the physical and everyday definitions of work. In everyday language, more work is considered to be done if the person becomes more tired afterward. Item 6 can most easily be answered by invoking the definition of work as the scalar product of force and displacement and realizing that because the angle between the force and displacement is $90^{\circ}$, the work must be zero. It can also be answered by using the work-kinetic energy theorem. Student response to item 6 shows that instruction was more successful in helping group $\mathrm{H}$ students understand the difference between velocity and speed than group L students. Item 8 assesses students' ability to differentiate between an applied force and the work done by it. Many incorrectly reasoned that because a smaller magnitude tension force is required along an inclined plane, the magnitude of the work done by tension or the gravitational force must therefore be smaller. The ability to distinguish between the force and the work done by the force did not improve at all post instruction for group L students. As mentioned earlier, part of the problem may be students' inability to grasp the distinction between the term work as used in physical and in everyday language. An interviewed student who chose (d) noted: ... you do a lot less work in the incline case than in the vertical case ... it is called mechanical advantage or something... When asked how he would calculate the work he added: ... I don't know if I remember the math ... the concept is clear to me though ... you definitely do less work when it is inclined. Another student had a similar explanation: in inclined case it would be easier to pull which implies less work for you. A third student explained: tension in case (ii) is at an angle ... say $30^{\circ}$ so the work done will have $\cos \left(30^{\circ}\right)$ and it will be smaller than straight up. Incidentally, 
the number of students from group $\mathrm{L}$ who believed that the work done by the gravitational force is path dependent in item 1 is significantly larger than in item 8 and this difference in number suggests that students' knowledge is fragmented and context-dependent. $\underline{1}$

Items $9,12,24$, and 25 probe the understanding of work done by non-conservative forces. In item 9, most of the improvement for group $\mathrm{H}$ from the pre-test to post-test was due to the transition from choice (b) to (a), because the students learned that a constant speed going up the hill does not imply that the mechanical energy is conserved. The trend is opposite for group L. A student who chose (e) explained: if you ignore the retarding effects of friction ... mechanical energy will be conserved no matter what. Other interviewed students who chose (e) also suggested that the retarding effect of friction was the only force that could change the mechanical energy of the system. Although some students may have chosen (b) because they could not distinguish between the kinetic and mechanical energies, the following interview excerpt shows why that option was chosen by a student despite the knowledge that kinetic and mechanical energies are different: if she goes up at constant speed then kinetic energy does not change ... that means potential energy does not change so the mechanical energy is conserved ... mechanical energy is kinetic plus potential. The student continued when asked to explain what the potential energy is: uhh ... isn't it mgh? When asked to explain why it is not changing, the student first paused and then added: ...h is the height ...I guess $h$ does change if she goes up the hill ...hmm ... may be that means that potential energy changes. I am confused ...I thought that if the kinetic energy does not change, then potential energy cannot change ... aren't the two supposed to compensate each other ... is it a realistic situation that she bikes up the hill at constant speed or is it just an ideal case? The student is convinced that the mechanical energy is conserved when the bike goes up at a constant speed, and he initially thinks that both the kinetic and potential energies must remain unchanged. When he confronts the fact that the potential energy is changing, instead of reasoning that the mechanical energy must be changing if the kinetic energy is constant, he thinks that it is probably not realistic to bike up the hill at a constant speed. He wonders if it is only possible in the idealized physics world. Although he ignores the work done by the non-conservative force applied on the pedal to keep the speed constant, his statements shed light on student's epistemological beliefs about how much one can trust physics to explain everyday phenomena. A student who chose (c) provided an interesting explanation: in case (1) the kinetic energy is transferred to potential energy so 
the mechanical energy is conserved and in case (2) ... obviously ... if the speed is constant ... mechanical energy is conserved. Case (3) is out because she is accelerating. This example shows student's inconsistent reasoning and use of the term acceleration. Actually, there is acceleration not only in case (3) but also in case (1) (slowing down), but the student ignores it in case (1). At the same time, he put cases (2) and (3) in different categories although the cyclist was pedaling in both cases. For item 12, the strongest distractor was the belief that there is a net force in the direction of motion. ${ }^{-1}$ This belief was essentially unchanged by instruction for group L while half of group $\mathrm{H}$ benefited from instruction. Items 24 and 25 can both be answered using the work-kinetic energy theorem. Item 24 has previously been investigated in depth, $\underset{2}{2}$ and students had great difficulty in realizing that because identical constant forces are applied over the same distance to both masses (which start from rest), their kinetic energies are identical regardless of their masses. Some students correctly stated that the velocity of block A will be greater, but they had difficulty in reasoning beyond this. Interviews show that the choice (b) was often dictated by the fact that the kinetic energy increases as the square of the speed but only linearly with mass. $\stackrel{2}{=}$ In item 25 , all distractors are dimensionally incorrect and equally popular.

Items 13 and 17 probe understanding of the conservation of mechanical energy and related issues. In item 13, some alternative choices used energy concepts while others used momentum concepts to assess how well students recognize the relevant concepts. In an interview, a student explained: (c) and (e) are obviously not right because they are the same ... momentum and kinetic energy both depend on mass and speed ... I think it is (b) because this one falls from a greater height. The student has forgotten about the vector character of momentum. Interviews show that at least some students who chose (a) were confident that the kinetic energy has a direction (is a vector) and the kinetic energies of the two carts cancel each other. In item 17, students who chose $(\mathrm{d})$ believed that the work done by the gravitational force on the ball falling from the tower is negative. Interviews show that many students did not invoke physics principles to come to this conclusion (for example, the basic definition of work), but thought that the work must be negative if the ball is falling in the "negative y direction." Students who focused on speed rather than kinetic energy were likely to get confused between options (a) and (b). An interviewed student who chose (a) started with the following correct statement: Isn't it true that the velocity of the ball increases by like $9.8 \mathrm{~m} / \mathrm{s}$ every second ... kinetic energy is $(1 / 2) m v^{2}$ (writes down the formula) . But later 
he got misled due to a faulty proportional reasoning and added referring to the formula: $v$ increases by equal amount over equal times... so $v^{2}$ increases by equal amount over equal times... mass $m$ is not changing...

Questions related to momentum concepts. Item 5 probes the understanding of momentum conservation in an elastic and inelastic collision. In item 5, students choosing the popular distractor (b) believed that the block in which the bullet gets embedded moves faster because the bullet transfers all of its kinetic energy to the block in the inelastic collision. The increased frequency of distractor (a) from pre-test to post-test shows that instruction was not effective in helping students learn that momentum is a vector quantity and when the bullet's direction changes during the elastic collision, it transfers momentum to the block. Another popular distractor (c), which has several buzz-words such as "Newton's second law" and "effective mass," is meaningless but was designed based upon student interviews.

Items 3 and 11 were designed partly to evaluate whether students can identify the appropriate system for which momentum (and also the kinetic energy for elastic collisions) is conserved and indicated that many students were confused about it. Many believed that the momentum and kinetic energy are conserved for each object. Incidentally, in the pre-test response to item 11, $48 \%$ noted that a person standing on ice will remain stationary when hit by a horizontally moving ball (regardless of whether it bounced elastically or not), because those choices had several buzz-words.

Items 10 and 21 probe understanding of momentum conservation in an inelastic collision or explosion. In item 10, many students believed that the kinetic energy is conserved in an explosion. Interviews show that some students believed that the kinetic energy is a vector. A student who chose (a) noted: it does not lose kinetic energy because there is no friction. Item 14 is about the comparison of two completely inelastic collisions, but the alternative choices included both the energy and momentum concepts and students had to determine which was appropriate. Even in item 21, different alternative choices included both momentum and mechanical energy conservation to assess if students could identify the relevant concepts. An interviewed student noted: I know this is an inelastic collision ... I will pick (a) because rain is falling vertically and is not going to influence the speed of the cart.... When asked about his thoughts on choice (c), the student paused and then added: ...probably the horizontal momentum is conserved but the rain did not have any horizontal momentum so the velocity of the cart in the horizontal direction is unchanged ...that's why I picked (a) ... The 
student's last statement is inconsistent because if the horizontal momentum is conserved, then the addition of mass should slow the cart. It is unclear however what the student meant by "the horizontal momentum is conserved." Taking item 3 as a guide, the student may very well be just referring to the cart as the system for which the horizontal momentum is conserved, although choice (c) explicitly noted the "cart-rain" system.

Items 19 and 23 are applications of the impulse-momentum theorem. In item 19, students had to realize that the change in momentum is the same whether the biker slams into a haystack or a concrete wall, but the haystack changes the momentum over a longer time and reduces the average force. In the interview, a student who chose (a) did not use the appropriate physics principle as the starting point and explained: . . haystack gives you a smaller force so the impulse is smaller. A student who chose (e) noted: ...harder objects give up more energy than softer objects. In item 23, the change in momentum of the rubber ball in the elastic collision with the surface is twice compared to that of the putty ball in the completely inelastic collision. Because the time $\Delta t$ over which the velocity of the balls changes from its initial to its final value due to the contact with the surface is the same, the average force on the surface by the rubber ball is twice as much as the putty ball. Interviews show that many students who chose distractor (a) reasoned that because the masses are the same, the force is proportional to the speed. Because both balls have the same speed when they hit the surface, the average force exerted on the surface is the same, regardless of whether the ball bounces back or gets stuck. Some students focused on the weights of the two balls and said that the balls would exert the same force on the surface because their weights were the same. Some said they did not know why $\Delta t$ was provided, because the weight does not depend upon time.

After instruction, students' overall performance improved on all test items (see gains in Table I) except item 16 on the ballistic pendulum, which was the only one that involved both the energy and momentum concepts simultaneously. Interviews show that some students who verbalized that the problem had several parts often ended up focusing on only one aspect. One student who chose (b) explained: because they are sticking ... it is a collision ... so momentum is conserved. Poor performance on this item, which involved both the energy and momentum concepts, suggests that even group $\mathrm{H}$ had difficulty analyzing multipart problems involving more than one concept ${ }^{11}$. In the post-test, student performance on items 7 and 18, which focused more on the definition and factual information (lower 
cognitive complexity), was above average. Responding to item 7, an interviewed student gave the following reasoning for why the motorcycle has a larger momentum: ... it has an acceleration which contributes to force which is involved in momentum.

\section{SUMMARY}

We have developed a research-based test to assess introductory students' conceptual understanding of energy and momentum. We found that students have difficulty in qualitatively interpreting the basic principles related to energy and momentum and in applying them in physical situations. The difficulties were not strongly dependent on student population or calculus background, except for honors and active-engagement classes. Comparison

of the pre-/post-test scores of students in the lower (L) and upper (H) quartiles based upon the overall test score shows that the upper-quartile group had significantly higher normalized gains. The test can be administered as a pre-/post-test to assess the difficulties prior to instruction, as well as those that remain uncorrected following a particular type of instructional intervention. The test can be used to compare the understanding of energy and momentum concepts in courses employing different instructional designs and strategies.

\section{Acknowledgments}

We are very grateful to all the faculty who reviewed the various components of the test at several stages and provided invaluable feedback. We are also very thankful to all the faculty who administered the test. This work is supported in part by the National Science Foundation and the Spencer Foundation.

\section{Appendix A: Energy and Momentum Conceptual Survey}

\section{Instructions}

- Select one of the five choices (a)-(e) for each of the 25 questions.

- Ignore the retarding effects of friction and air resistance unless otherwise stated.

1. You lift a suitcase from the floor to a table. In addition to the weight of the suitcase, 
select all of the following factors that determine the work done by the gravitational force on the suitcase.

(1) whether you lift it directly up to the table or along a longer path

(2) whether you lift it quickly or slowly

(3) the height of the table above the floor

(a) (1) only

(b) (3) only

(c) (1) and (3) only

(d) (2) and (3) only

(e) (1), (2) and (3)

2. Two frictionless slides are shaped differently but start at the same height $h$ and end at the same level as shown below. You and your friend, who has the same weight as you, slide down from the top on different slides starting from rest. Which one of the following statements best describes who has a larger speed at the bottom of the slide?

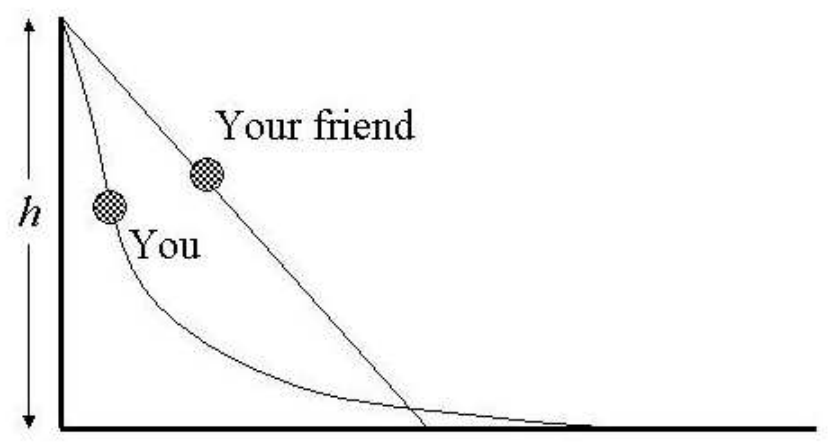

(1) You, because you initially encounter a steeper slope so that there is more opportunity for accelerating.

(2) You, because you travel a longer distance so that there is more opportunity for accelerating.

(3) Your friend, because her slide has a constant slope so that she has more opportunity for accelerating. 
(4) Your friend, because she travels a shorter distance so that she can conserve her kinetic energy better.

(5) Both of you have the same speed.

3. A moving white hockey puck collides elastically with a stationary red hockey puck on a frictionless horizontal surface. No net external force acts on the two-puck system. Select all of the following statements that must be true for this elastic collision.

(1) The kinetic energy of the white puck is conserved (same before and after the collision).

(2) The linear momentum of the white puck is conserved.

(3) The linear momentum of the two-puck system is conserved.

(a) (1) only

(b) (3) only

(c) (1) and (2) only

(d) (1) and (3) only

(e) (1), (2) and (3)

4. Two identical stones, A and B, are shot from a cliff from the same height and with identical initial speeds $v_{0}$. Stone A is shot vertically up, and stone B is shot vertically down (see Figure). Which one of the following statements best describes which stone has a larger speed right before it hits the ground
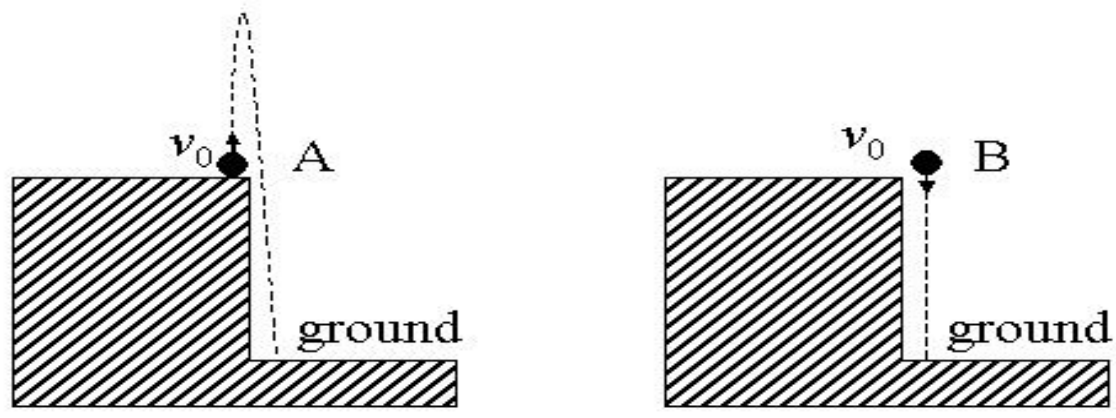

(a) Both stones have the same speed. 
(b) A, because it travels a longer path.

(c) A, because it takes a longer time.

(d) A, because it travels a longer path and takes a longer time.

(e) $\mathrm{B}$, because no work is done against the gravitational force.

5. Two identical bullets are fired horizontally with identical speeds $v_{0}$ at two blocks of equal mass. The blocks rest on a frictionless horizontal surface and are made of hard steel and soft wood respectively (see Figure). One bullet bounces elastically off the steel block. The other bullet becomes embedded inside the wood block. Which one of the following statements best describes which block travels faster after the collision?

\section{TOP VIEW}
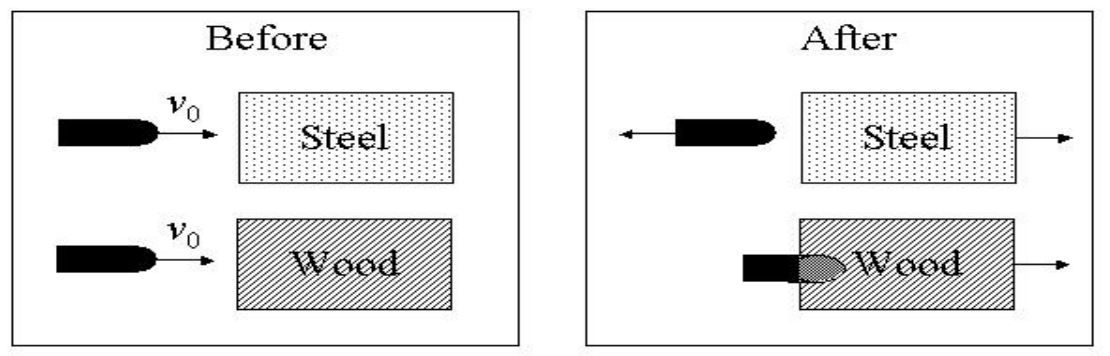

(a) The wood block, because it has gained the momentum of the bullet, while the other bullet does not impart its momentum to the steel block.

(b) The wood block, because the bullet transfers all of its kinetic energy to it.

(c) The wood block, because its larger effective mass after the collision, in accordance with Newton's second law, results in a larger force to accelerate the block.

(d) The steel block, because the bullet bounces off from it.

(e) Both blocks travel with the same speed.

6. A satellite is moving around Earth in a circular orbit at a constant speed (see Figure). The only force that acts on the satellite is Earth's gravitational force which points directly toward earth's center. Which one of the following statements is true as the satellite moves from point A to point B in the orbit? 


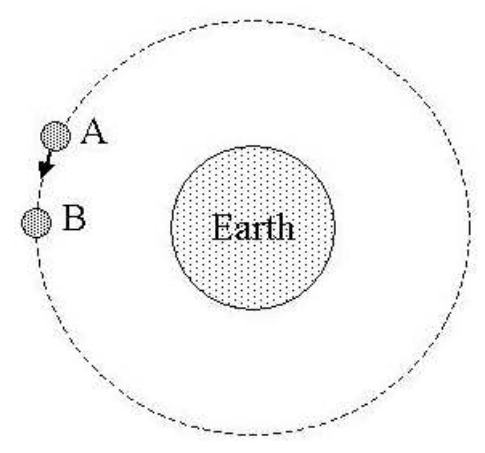

(a) The gravitational potential energy of the satellite decreases as it moves from A to B.

(b) The work done on the satellite by the gravitational force is negative for the motion from $\mathrm{A}$ to $\mathrm{B}$.

(c) The work done on the satellite by the gravitational force is zero for the motion from A to B.

(d) The velocity of the satellite remains unchanged as it moves from A to B.

(e) None of the above.

7. A motorcycle and a truck are both moving in the same direction in adjacent lanes on a highway. At a particular instant, the speed of the motorcycle is four times as large as the speed of the truck $v$. At that instant, the motorcycle is accelerating forward while the truck is moving at a constant velocity. Which one of the following statements best describes which vehicle has a larger momentum at that instant?

\begin{tabular}{|c|c|c|}
\hline Hare & $\stackrel{v}{*}$ & Acceleration $\alpha=0$ \\
\hline motorcycle & $4 v$ & \multirow{2}{*}{ Acceleration $a \neq 0$} \\
\hline ৃ: & & \\
\hline
\end{tabular}

(a) The truck, because it has a larger mass.

(b) The motorcycle, because it is moving faster.

(c) The motorcycle, because it has an acceleration. 
(d) The motorcycle, because it is moving faster and also has an acceleration.

(e) Not enough information.

8. You want to lift a heavy block through a height $h$ by attaching a string of negligible mass to it and pulling so that it moves at a constant velocity. You have the choice of lifting it either by pulling the string vertically upward or along a frictionless inclined plane (see Figure). Which one of the following statements is true?

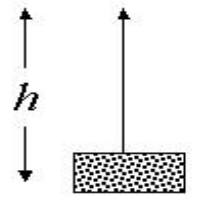

(i)

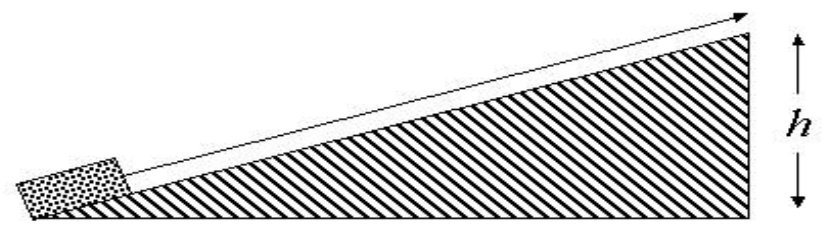

(ii)

(a) The magnitude of the tension force in the string is smaller in case (i) than in case (ii).

(b) The magnitude of the tension force in the string is the same in both cases.

(c) The work done on the block by the tension force is the same in both cases.

(d) The work done on the block by the tension force is smaller in case (ii) than in case (i).

(e) The work done on the block by the gravitational force is smaller in case (ii) than in case (i).

9. Three bicycles approach a hill as described below:

(1) Cyclist 1 stops pedaling at the bottom of the hill, and her bicycle coasts up the hill.

(2) Cyclist 2 pedals so that her bicycle goes up the hill at a constant speed.

(3) Cyclist 3 pedals harder, so that her bicycle accelerates up the hill.

Ignoring the retarding effects of friction, select all the cases in which the total mechanical energy of the cyclist and bicycle is conserved. 
(a) (1) only

(b) (2) only

(c) (1) and (2) only

(d) (2) and (3) only

(e) (1), (2) and (3)

10. A bomb at rest on a horizontal frictionless surface explodes and breaks into three pieces that fly apart horizontally as shown below. Select all of the following statements that must be true after the bomb has exploded.

(1) The total kinetic energy of the bomb fragments is the same as that of the bomb before explosion.

(2) The total momentum of the bomb fragments is the same as that of the bomb before explosion.

(3) The total momentum of the bomb fragments is zero.

\section{TOP VIEW}
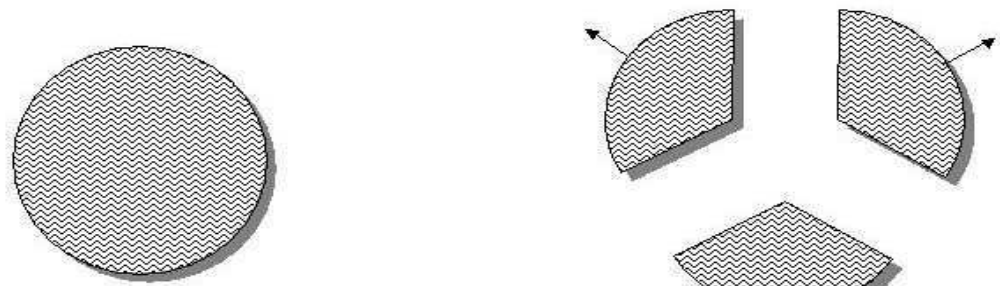

Before

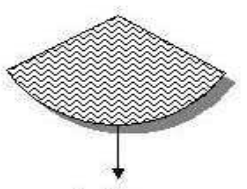

After

(a) (1) only

(b) (2) only

(c) (3) only

(d) (2) and (3) only

(e) (1), (2) and (3) 
11. You and your friend are both standing on a horizontal frictionless surface. To get your friend's attention, you throw a ball due west at your friend as shown below. The ball bounces elastically off your friend's back. Which one of the following statements is true about this situation?

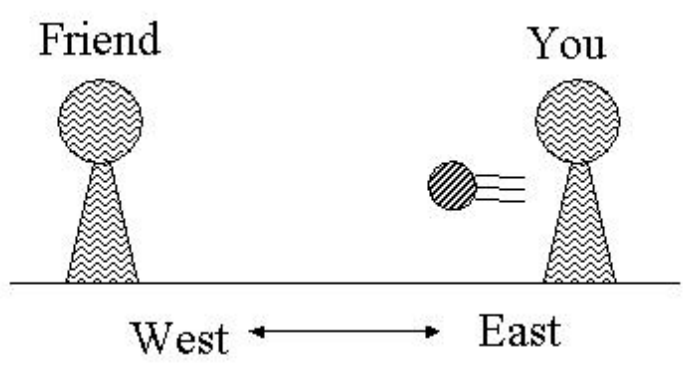

(a) Your friend will remain stationary because the ball bounces elastically and does not impart its momentum to her.

(b) Your friend will remain stationary because the kinetic energy of the ball is conserved in an elastic collision.

(c) Your friend will remain stationary due to conservation of both linear momentum and kinetic energy.

(d) You will remain stationary after you throw the ball due to conservation of both linear momentum and kinetic energy.

(e) You will move east after you throw the ball due to conservation of linear momentum.

12. Using a rope of negligible mass, you pull a box along a horizontal surface with a constant horizontal force $\mathbf{F}_{\mathbf{A}}$. The box moves at a constant velocity from position A to position B. The force of friction $\mathbf{F}_{\mathbf{k}}$ cannot be neglected. Which one of the following statements concerning the motion of the box from A to B is true?

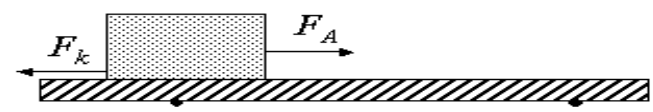

A Before

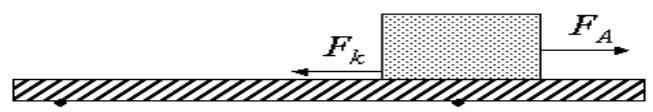

A

$\mathrm{B}$

After

(a) The work done on the box by the gravitational force is non-zero. 
(b) The work done on the box by $\mathbf{F}_{\mathbf{k}}$ is positive.

(c) The total work done on the box by the net force is non-zero.

(d) The magnitude of the work done on the box by $\mathbf{F}_{\mathbf{A}}$ is equal to the magnitude of the work done by $\mathbf{F}_{\mathbf{k}}$.

(e) The magnitude of $\mathbf{F}_{\mathbf{A}}$ is greater than the magnitude of $\mathbf{F}_{\mathbf{k}}$.

\section{- Setup for Questions (13) and (14)}

Carts $\mathrm{A}$ and $\mathrm{B}$ are identical in all respects before the collision.

Figure (i): Cart A starts from rest on a hill at a height $h$ above the ground. It rolls down and collides "head-on" with cart B which is initially at rest on the ground. The two carts stick together.

Figure (ii): Carts $\mathrm{A}$ and $\mathrm{B}$ are at rest on opposite hills at heights $h / 2$ above the ground. They roll down, collide "head-on" with each other on the ground and stick together.

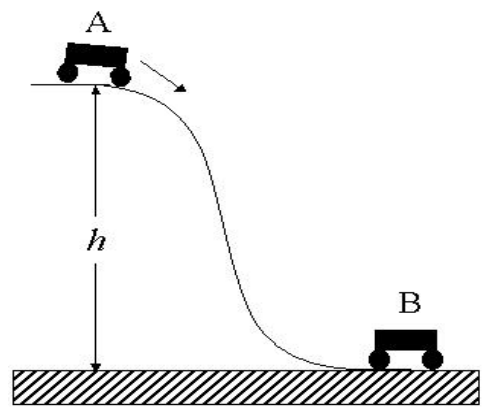

(i)

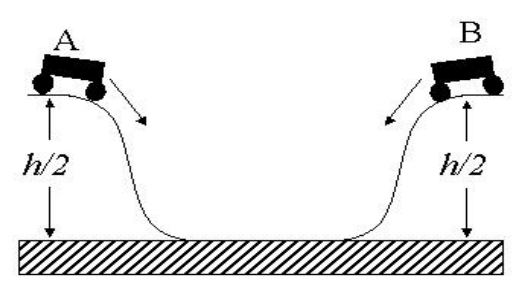

(ii)

13. Which one of the following statements is true about the two-cart system just before the carts collide in the two cases?

Just before the collision on the ground,

(a) the kinetic energy of the system is zero in case (ii).

(b) the kinetic energy of the system is greater in case (i) than in case (ii). 
(c) the kinetic energy of the system is the same in both cases.

(d) the momentum of the system is greater in case (ii) than in case (i).

(e) the momentum of the system is the same in both cases.

14. Which one of the following statements is true about the two-cart system just after the carts collide in the two cases?

Just after the collision,

(a) the kinetic energy of the system is greater in case (ii) than in case (i).

(b) the kinetic energy of the system is the same in both cases.

(c) the momentum of the system is greater in case (ii) than in case (i).

(d) the momentum of the system is non-zero in case (i) while it is zero in case (ii).

(e) the momentum of the system is the same in both cases.

15. While in a playground, you and your niece take turns sliding down a frictionless slide. Your mass is $75 \mathrm{~kg}$ while your little niece's mass is only $25 \mathrm{~kg}$. Assume that both of you begin sliding from rest from the same height. Which one of the following statements best describes who has a larger speed at the bottom of the slide?

(a) Both of you have the same speed at the bottom.

(b) Your niece, because she is not pressing down against the slide as strongly so her motion is closer to free fall than yours.

(c) You, because your greater weight causes a greater downward acceleration.

(d) Your niece, because lighter objects are easier to accelerate.

(e) You, because you take less time to slide down.

16. Two small spheres of putty, A and B, of equal mass, hang from the ceiling on massless strings of equal length. Sphere A is raised to a height $h_{0}$ as shown below and released. It collides with sphere B (which is initially at rest); they stick and swing together to a maximum height $h_{f}$. The height $h_{f}$ can be determined in terms of $h_{0}$ by invoking which of the following principles: 
(1) the conservation of mechanical energy

(2) the conservation of linear momentum

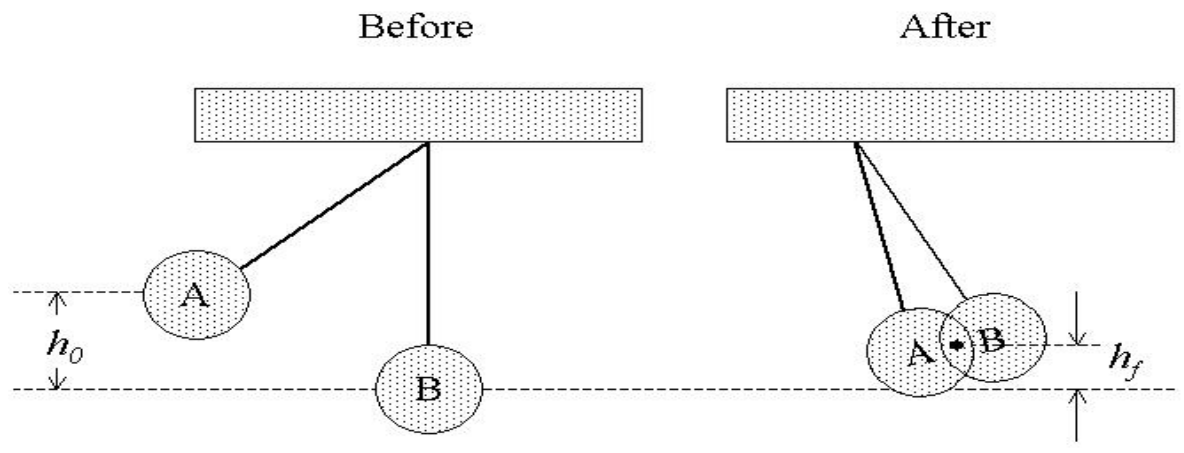

(a) (1) only

(b) (2) only

(c) both (1) and (2)

(d) either (1) or (2) but not both

(e) Principles (1) and (2) alone are not sufficient to find $h_{f}$ in terms of $h_{0}$.

17. You drop a ball from a high tower and it falls freely under the influence of the gravitational force. Which one of the following statements is true?

(a) The kinetic energy of the ball increases by equal amounts in equal times.

(b) The kinetic energy of the ball increases by equal amounts over equal distances.

(c) There is zero work done on the ball by the gravitational force as it falls.

(d) The work done on the ball by the gravitational force is negative as it falls.

(e) The total mechanical energy of the ball decreases as it falls.

- In Questions (18) and (19) below, the impulse of a force is defined as the product of the average force and the time interval during which the force acts.

18. Which one of the following statements is true concerning linear momentum?

(a) Momentum is a force. 
(b) The momentum of an object is always positive.

(c) Momentum is a scalar quantity.

(d) The SI unit of momentum is $\mathrm{kg} \mathrm{m}^{2} / \mathrm{s}$.

(e) Momentum and impulse have the same units.

19. The brakes of your bicycle have failed, and you must choose between slamming into either a haystack or a concrete wall. Which one of the following statements best justifies why hitting a haystack is a wiser choice than hitting a concrete wall?

(a) The haystack gives you a smaller impulse than the concrete wall.

(b) The haystack changes your momentum over a longer time.

(c) Your change in kinetic energy is smaller if you hit the haystack than if you hit the concrete wall.

(d) Your change in momentum is smaller if you hit the haystack than if you hit the concrete wall.

(e) More potential energy is stored in the wall which is released upon the impact and results in a greater force on you.

20. You slide down two consecutive slopes of frictionless ice whose vertical heights $h$ are identical, as shown below. Select all of the following statements that must be true.

(1) The change in your kinetic energy is identical for the motion from A to B and from B to C.

(2) The work done on you by the gravitational force is smaller for the motion from A to B than from B to C.

(3) The work done on you by the gravitational force is greater for the motion from A to B than from B to C.

(a) (1) only

(b) (2) only

(c) (3) only 
(d) (1) and (2) only

(e) (1) and (3) only

21. Rain starts falling vertically down into a cart with frictionless wheels which is initially moving at a constant velocity on a horizontal surface. The rain drops come to rest with respect to the cart after striking it, and rain water accumulates in the cart. Select all of the following statements that must be true about this situation.

(1) The cart will continue to move at a constant velocity because the rain is falling vertically while the cart is moving horizontally.

(2) The cart will continue to move at a constant velocity because the total mechanical energy of the cart-rain system is conserved.

(3) The cart will slow down because the horizontal momentum of the cart-rain system is conserved.

(a) (1) only

(b) (2) only

(c) (3) only

(d) (1) and (2) only

(e) None of the above

22. Three balls are launched from the same horizontal level with identical speeds $v_{0}$ as shown below. Ball (1) is launched vertically upward, ball (2) at an angle of $60^{\circ}$, and ball (3) at an angle of $45^{\circ}$. In order of decreasing speed (fastest first), rank the speed each one attains when it reaches the level of the dashed horizontal line. All three balls have sufficient speed to reach the dashed line.

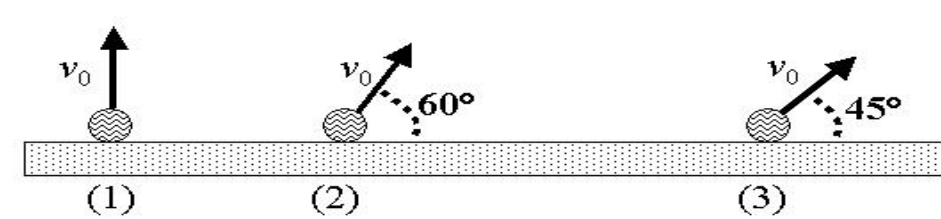


(a) (1), (2), (3)

(b) (1), (3), (2)

(c) (3), (2), (1)

(d) They all have the same speed.

(e) Not enough information, their speeds will depend on their masses.

23. You drop two balls of equal mass, made of rubber and putty, from the same height $h$ above a horizontal surface (see Figure). The rubber ball bounces up after it strikes the surface while the putty ball comes to rest after striking it. Assume that in both cases the velocity of the ball takes the same time $\Delta t$ to change from its initial to its final value due to contact with the surface. Compare the average forces $\overline{\mathbf{F}}_{R}$ and $\overline{\mathbf{F}}_{P}$ exerted on the surface by the rubber and putty balls, respectively, during time $\Delta t$.

\section{$\underline{\text { Rubber }}$}

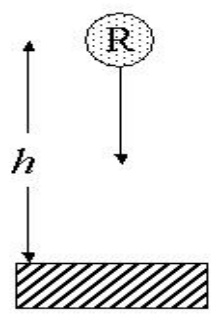

Before
After

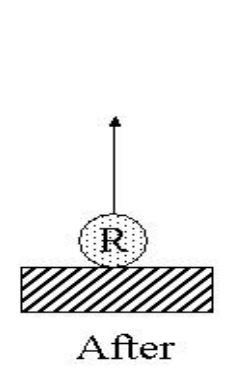

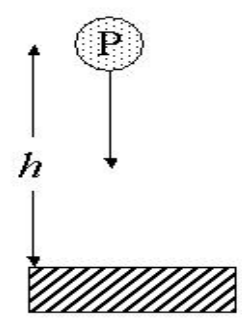

Before
Putty

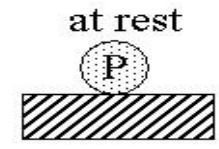

After

(a) $\overline{\mathbf{F}}_{R}=\overline{\mathbf{F}}_{P}$

(b) $\overline{\mathbf{F}}_{R}>\overline{\mathbf{F}}_{P}$

(c) $\overline{\mathbf{F}}_{R}<\overline{\mathbf{F}}_{P}$

(d) $\overline{\mathbf{F}}_{R}$ may be smaller or larger than $\overline{\mathbf{F}}_{P}$ depending upon the relative size of the balls.

(e) $\overline{\mathbf{F}}_{R}$ may be smaller or larger than $\overline{\mathbf{F}}_{P}$ depending upon the actual height $h$ from which the balls are dropped.

24. Two blocks are initially at rest on a frictionless horizontal surface. The mass $m_{A}$ of block $\mathrm{A}$ is less than the mass $m_{B}$ of block B. You apply the same constant force $\mathbf{F}$ and pull the blocks through the same distance $d$ along a straight line as shown below (force $\mathbf{F}$ is applied for the entire distance $d$ ). 


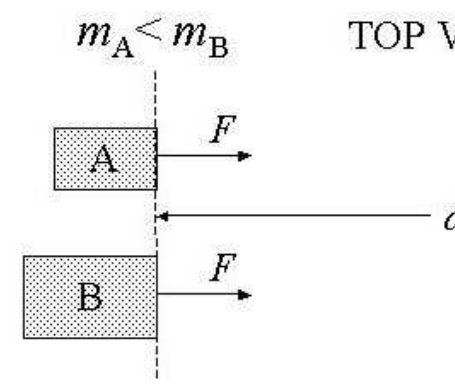

START

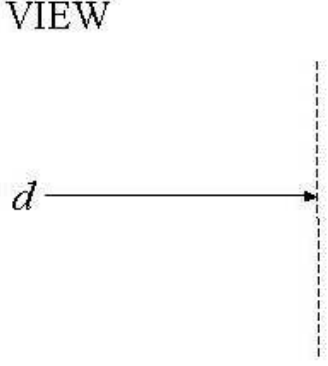

FINISH

Which one of the following statements correctly compares the kinetic energies of the blocks after you pull them the same distance $d$ ?

(a) The kinetic energies of both blocks are identical.

(b) The kinetic energy is greater for the smaller mass block because it achieves a larger speed.

(c) The kinetic energy is greater for the larger mass block because of its larger mass.

(d) Not enough information, need to know the actual mass of both blocks to compare the kinetic energies.

(e) Not enough information, need to know the actual magnitude of force $\mathbf{F}$ to compare the kinetic energies.

25. A box slides with an initial speed $v_{o}$ on a horizontal surface with friction and eventually comes to a stop. Which one of the following is equal to the change in the kinetic energy of the box?

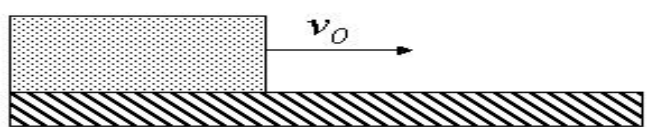

Before

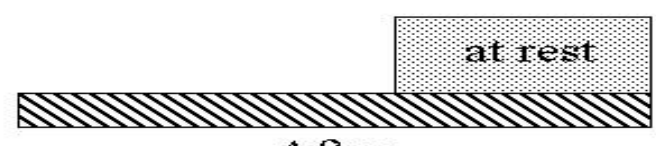

After

(a) The momentum of the box multiplied by the distance travelled before coming to rest. 
(b) The momentum of the box multiplied by the time elapsed before coming to rest.

(c) The momentum of the box multiplied by the deceleration of the box.

(d) The mass of the box multiplied by the deceleration of the box.

(e) None of the above. 
1 D. Hestenes, M. Wells, and G. Swackhamer, "Force concept inventory," Phys. Teach. 30, 141151 (1992); R. K. Thornton and D. R. Sokoloff, “Assessing student learning of Newton's laws: The force and motion conceptual evaluation and the evaluation of active learning laboratory and lecture curricula," Am. J. Phys. 66, 338-352 (1998); R. J. Beichner, "Testing student interpretation of kinematics graphs," Am. J. Phys. 62, 750-762 (1994); R. Thornton, "Questions on heat and temperature," Unpublished, (1992); D. R. Sokoloff, "Electric circuit concept test," Unpublished, (1993); D. P. Maloney, T. L. O'Kuma, C. J. Hieggelke, and A. V. Heuvelen, "Surveying students' conceptual knowledge of electricity and magnetism," Phys. Ed. Res., Am. J. Phys. 69(7), S12-S23, (2001).

2 A. B. Arons, A Guide to Introductory Physics Teaching (Wiley, NY, 1990); B. A. Sherwood and W. H. Bernard, "Work and heat transfer in the presence of sliding friction," Am. J. Phys. 52, 1001-1007 (1984); A. Van Heuvelen and X. Zou, "Multiple representations of work-energy processes," Am. J. Phys. 69(2), 184-194 (2001); R. A. Lawson and L. C. McDermott, "Student understanding of the work-energy and impulse-momentum theorems," Am. J. Phys. 55(9), 811817 (1987); T. O’Brien Pride, S. Vokos, and L. C. McDermott, "The challenge of matching learning assessments to teaching goals: An example from the work-energy and impulse-momentum theorems," Am. J. Phys. 66 (2), 147-157 (1998).

3 C. Singh and D. Rosengrant, "Students' conceptual knowledge of energy and momentum," Proceedings of the Physics Education Research conference, (Rochester, NY), edited by S. Franklin, J. Marx, and K. Cummings, 123, 2001.

4 G. Aubrecht and J. Aubrecht, "Constructing objective tests," Am. J. Phys. 51, 613-620 (1983); A. J. Nitko, Educational Assessments of Students (Prentice Hall/Merrill, Englewood Cliffs, NJ, 1996), 2nd ed.

5 B. Bloom, Taxonomy of Educational Objectives (Longman, New York, 1987).

6 M. Chi, "Thinking aloud," in The Think Aloud Method: A Practical Guide to Modeling Cognitive Processes, edited by M. W. Van Someren, Y. F. Barnard, and J. A. C. Sandberg (Academic Press, London, 1994) Chap. 1.

7 R. Gorsuch, Factor Analysis (Lawrence Erlbaum, Hillsdale, NJ, 1983).

8 J. Cohen, Statistical Power Analysis for the Behavioral Sciences (Academic Press, NY, 1969). 
9 R. Hake, "Interactive-engagement vs. traditional methods: A six-thousand-student survey of mechanics test data for introductory physics courses," Am. J. Phys. 66, 64-74 (1998).

10 M. T. H. Chi, P. J. Feltovich, and R. Glaser, "Categorization and representation of physics knowledge by expert and novices", Cog. Sci. 5, 121-152 (1981).

11 C. Singh, "When physical intuition fails", Am. J. Phys, 70(11), 1103-1109, (2002). 


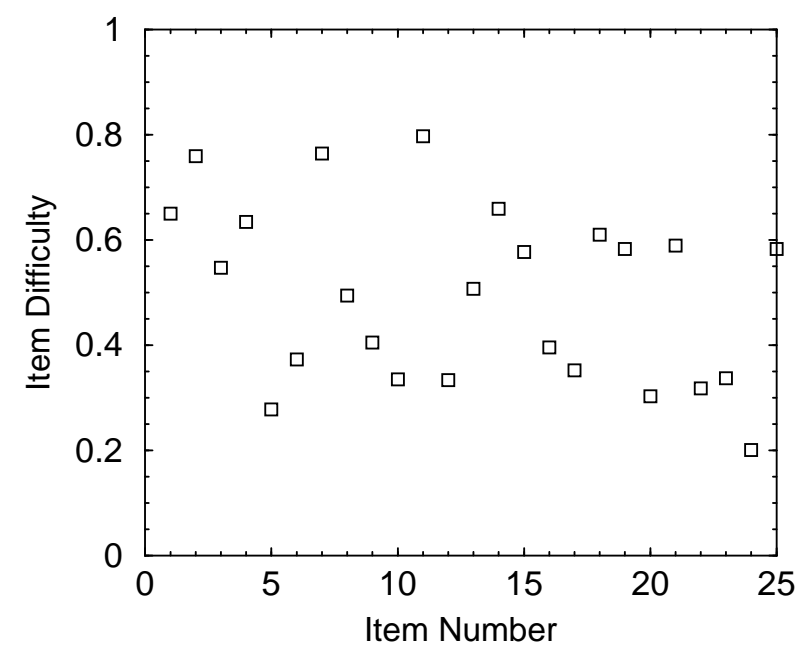

FIG. 1: Item difficulty (fraction correct) for each item on the test for 1170 students

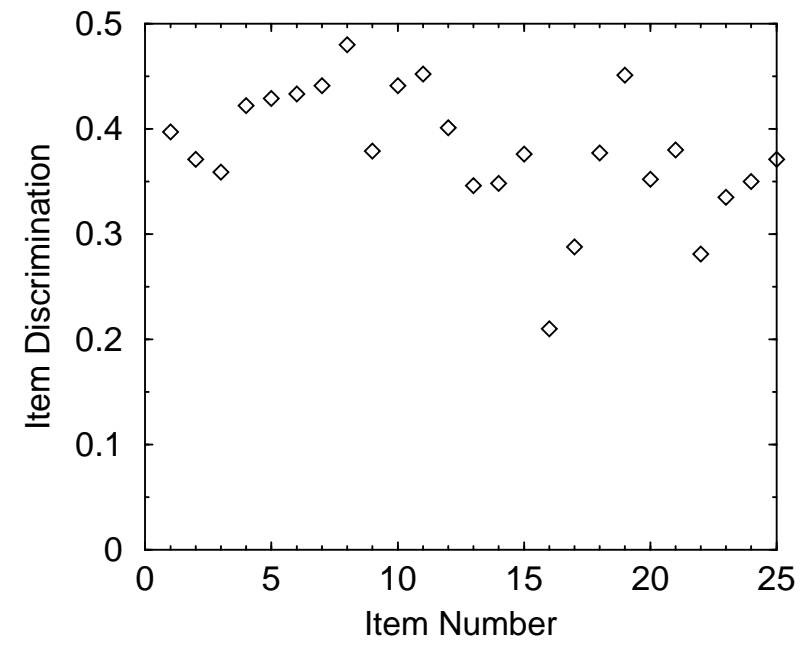

FIG. 2: Item discrimination for each item on the test for 1170 students 


\begin{tabular}{|c|c|c|c|c|c|c|c|c|c|}
\hline Item \# & Pre-H & Post-H & Pre-L & Post-L & Pre-A & Post-A & $g_{H}$ & $g_{L}$ & $g_{A}$ \\
\hline 1 & 57 & 82 & 14 & 36 & 33 & 63 & 0.57 & 0.25 & 0.45 \\
\hline 2 & 78 & 97 & 25 & 59 & 50 & 81 & 0.86 & 0.45 & 0.63 \\
\hline 3 & 66 & 82 & 24 & 36 & 45 & 61 & 0.47 & 0.15 & 0.30 \\
\hline 4 & 68 & 89 & 31 & 42 & 47 & 69 & 0.65 & 0.16 & 0.40 \\
\hline 5 & 18 & 51 & 4 & 7 & 11 & 27 & 0.40 & 0.04 & 0.18 \\
\hline 6 & 19 & 52 & 11 & 16 & 12 & 28 & 0.40 & 0.06 & 0.18 \\
\hline 7 & 75 & 97 & 24 & 62 & 55 & 85 & 0.88 & 0.50 & 0.66 \\
\hline 8 & 54 & 70 & 14 & 13 & 30 & 45 & 0.35 & -0.02 & 0.22 \\
\hline 9 & 36 & 61 & 27 & 18 & 26 & 36 & 0.38 & -0.12 & 0.13 \\
\hline 10 & 22 & 57 & 5 & 15 & 13 & 35 & 0.44 & 0.10 & 0.25 \\
\hline 11 & 68 & 95 & 14 & 56 & 41 & 79 & 0.84 & 0.48 & 0.65 \\
\hline 12 & 34 & 57 & 13 & 17 & 21 & 33 & 0.34 & 0.04 & 0.15 \\
\hline 13 & 69 & 75 & 28 & 35 & 52 & 53 & 0.18 & 0.10 & 0.03 \\
\hline 14 & 78 & 89 & 37 & 52 & 60 & 70 & 0.49 & 0.24 & 0.25 \\
\hline 15 & 74 & 80 & 24 & 40 & 47 & 58 & 0.23 & 0.21 & 0.22 \\
\hline 16 & 41 & 36 & 29 & 17 & 34 & 26 & -0.08 & -0.18 & -0.11 \\
\hline 17 & 34 & 60 & 23 & 32 & 30 & 46 & 0.39 & 0.11 & 0.23 \\
\hline 18 & 32 & 84 & 12 & 50 & 20 & 66 & 0.76 & 0.43 & 0.57 \\
\hline 19 & 71 & 82 & 22 & 52 & 47 & 70 & 0.37 & 0.39 & 0.43 \\
\hline 20 & 62 & 80 & 11 & 30 & 35 & 58 & 0.46 & 0.21 & 0.34 \\
\hline 21 & 51 & 83 & 36 & 42 & 44 & 62 & 0.65 & 0.10 & 0.32 \\
\hline 22 & 28 & 48 & 6 & 7 & 16 & 29 & 0.27 & 0.01 & 0.16 \\
\hline 23 & 27 & 41 & 16 & 18 & 21 & 25 & 0.20 & 0.02 & 0.06 \\
\hline 24 & 34 & 47 & 6 & 9 & 20 & 29 & 0.19 & 0.03 & 0.11 \\
\hline 25 & 47 & 75 & 20 & 44 & 33 & 59 & 0.52 & 0.30 & 0.39 \\
\hline
\end{tabular}

TABLE I: Student performance before and after instruction. The average percent pre-/post-test scores and normalized gain $g$ on each item for the upper $25 \%(\mathrm{H})$, Lower $25 \%(\mathrm{~L})$, and for all students (A). 


\begin{tabular}{|c|c|c|c|c|c|c|c|c|c|c|c|c|c|c|c|c|c|c|c|c|}
\hline \multirow{2}{*}{$\begin{array}{c}\text { Item } \\
\#\end{array}$} & \multicolumn{5}{|c|}{ pre-test (L) } & \multicolumn{5}{|c|}{ post-test (L) } & \multicolumn{5}{|c|}{ pre-test (A) } & \multicolumn{5}{|c|}{ post-test (A) } \\
\hline & $\mathrm{a}$ & b & $\mathrm{c}$ & $\mathrm{d}$ & $\mathrm{e}$ & $\mathrm{a}$ & $\mathrm{b}$ & c & d & e & & $\mathrm{b}$ & c & d & $\mathrm{e}$ & $\mathrm{a}$ & $\mathrm{b}$ & $\mathrm{c}$ & d & \\
\hline 1 & 13 & 14 & 47 & 13 & 13 & 6 & 36 & 39 & 6 & 13 & 10 & 33 & 37 & 9 & 11 & 4 & 63 & 21 & 6 & 6 \\
\hline 2 & 27 & 8 & & r & 25 & 17 & 4 & 13 & 7 & 59 & 15 & 7 & 21 & 7 & 50 & 7 & 2 & 6 & 4 & 81 \\
\hline 3 & 21 & 24 & 24 & 19 & 12 & 5 & 36 & 8 & 32 & 19 & 13 & 45 & 16 & 16 & 10 & 3 & 61 & 3 & 21 & 12 \\
\hline 4 & 31 & 14 & & 23 & 28 & 42 & 10 & 4 & 18 & 26 & 47 & 14 & 3 & 15 & 21 & 69 & 9 & 3 & 8 & 11 \\
\hline 5 & 23 & 41 & 21 & 4 & 11 & 32 & 33 & 19 & 7 & 9 & 15 & 45 & 17 & 11 & 12 & 20 & 30 & 12 & 27 & 11 \\
\hline 6 & 4 & 7 & 1 & 65 & 13 & 5 & 6 & 16 & 55 & 18 & 5 & 4 & 12 & 66 & 13 & 3 & 8 & 28 & 37 & 24 \\
\hline 7 & 21 & 5 & 19 & 31 & 24 & 11 & 14 & 4 & 9 & 62 & 15 & 5 & 10 & 15 & 55 & 5 & 4 & 2 & 4 & 85 \\
\hline 8 & 8 & 0 & 14 & 48 & 21 & 12 & 8 & 13 & 52 & 15 & 6 & 8 & 30 & 41 & 15 & 6 & 6 & 45 & 33 & 10 \\
\hline 9 & 27 & 27 & 23 & 17 & 6 & 18 & 34 & 18 & 9 & 21 & 26 & 33 & 14 & 12 & 15 & 36 & 24 & 12 & 4 & 24 \\
\hline 10 & 44 & 21 & 1 & & 11 & 20 & 24 & 27 & 15 & 14 & 35 & 20 & 21 & 13 & 11 & 14 & 14 & 18 & 35 & 19 \\
\hline 11 & 16 & 33 & 19 & 18 & 14 & 4 & 12 & 12 & 16 & 56 & 13 & 23 & 12 & 11 & 41 & 3 & 6 & 6 & 6 & 79 \\
\hline 12 & 6 & & & 13 & 64 & 7 & 3 & 13 & 17 & 60 & 5 & & 9 & 21 & 61 & 2 & 2 & 12 & 33 & 51 \\
\hline 13 & 7 & 32 & 28 & 18 & 15 & 11 & 24 & 35 & 15 & 15 & 6 & 18 & 52 & 10 & 14 & 7 & 14 & 53 & 9 & 17 \\
\hline 14 & 14 & 18 & 18 & 37 & 13 & 11 & 14 & 5 & 52 & 18 & 6 & 14 & 8 & 60 & 12 & 5 & 7 & 3 & 70 & 15 \\
\hline 15 & 24 & 10 & 55 & 6 & 5 & 40 & 6 & 42 & 9 & 3 & 47 & 6 & 39 & 4 & 4 & 58 & 2 & 31 & 5 & 4 \\
\hline 16 & 20 & 29 & 29 & 14 & 8 & 37 & 24 & 17 & 9 & 13 & 24 & 22 & 34 & 12 & 8 & 37 & 19 & 26 & 6 & 12 \\
\hline 17 & 37 & 23 & 7 & 26 & 7 & 28 & 32 & 5 & 31 & 4 & 31 & 30 & 5 & 28 & 6 & 27 & 46 & 2 & 23 & 2 \\
\hline 18 & 26 & 19 & 20 & 23 & 12 & 12 & 7 & 23 & 8 & 50 & 23 & 17 & 19 & 21 & 20 & 9 & 5 & 15 & 5 & 66 \\
\hline 19 & 14 & 22 & 11 & 20 & 33 & 24 & 52 & 7 & 10 & 7 & 14 & 47 & 7 & 14 & 18 & 19 & 70 & 3 & 4 & 4 \\
\hline 20 & 11 & 13 & 48 & 12 & 16 & 30 & 12 & 28 & 15 & 15 & 35 & 11 & 33 & 8 & 13 & 58 & 7 & 12 & 11 & 12 \\
\hline 21 & 8 & 23 & 36 & 11 & 22 & 15 & 8 & 42 & 19 & 16 & 13 & 15 & 44 & 11 & 17 & 10 & 5 & 62 & 11 & 12 \\
\hline 22 & 36 & 11 & 24 & 6 & 23 & 50 & 10 & 23 & 7 & 10 & 35 & 7 & 21 & 16 & 21 & 39 & 5 & 16 & 29 & 11 \\
\hline 23 & 32 & 16 & 28 & 19 & 5 & 43 & 18 & 29 & 6 & 4 & 45 & 21 & 20 & 9 & 5 & 50 & 25 & 19 & 4 & 2 \\
\hline 24 & 6 & & 34 & 18 & 7 & 9 & 27 & $37 \mid$ & 14 & 13 & 20 & 29 & 29 & 15 & 7 & 29 & 24 & 21 & 20 & 6 \\
\hline 25 & 20 & & 8 & 19 & 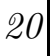 & 13 & 12 & 16 & 15 & 44 & 15 & 13 & 19 & 20 & 33 & 8 & 9 & 12 & & 59 \\
\hline
\end{tabular}

TABLE II: Distribution of alternative choices (correct choices in italics). The percent from lower $25 \%$ (L) and percent of all students (A) who chose options (a)-(e) in the pre-test and post-test. 


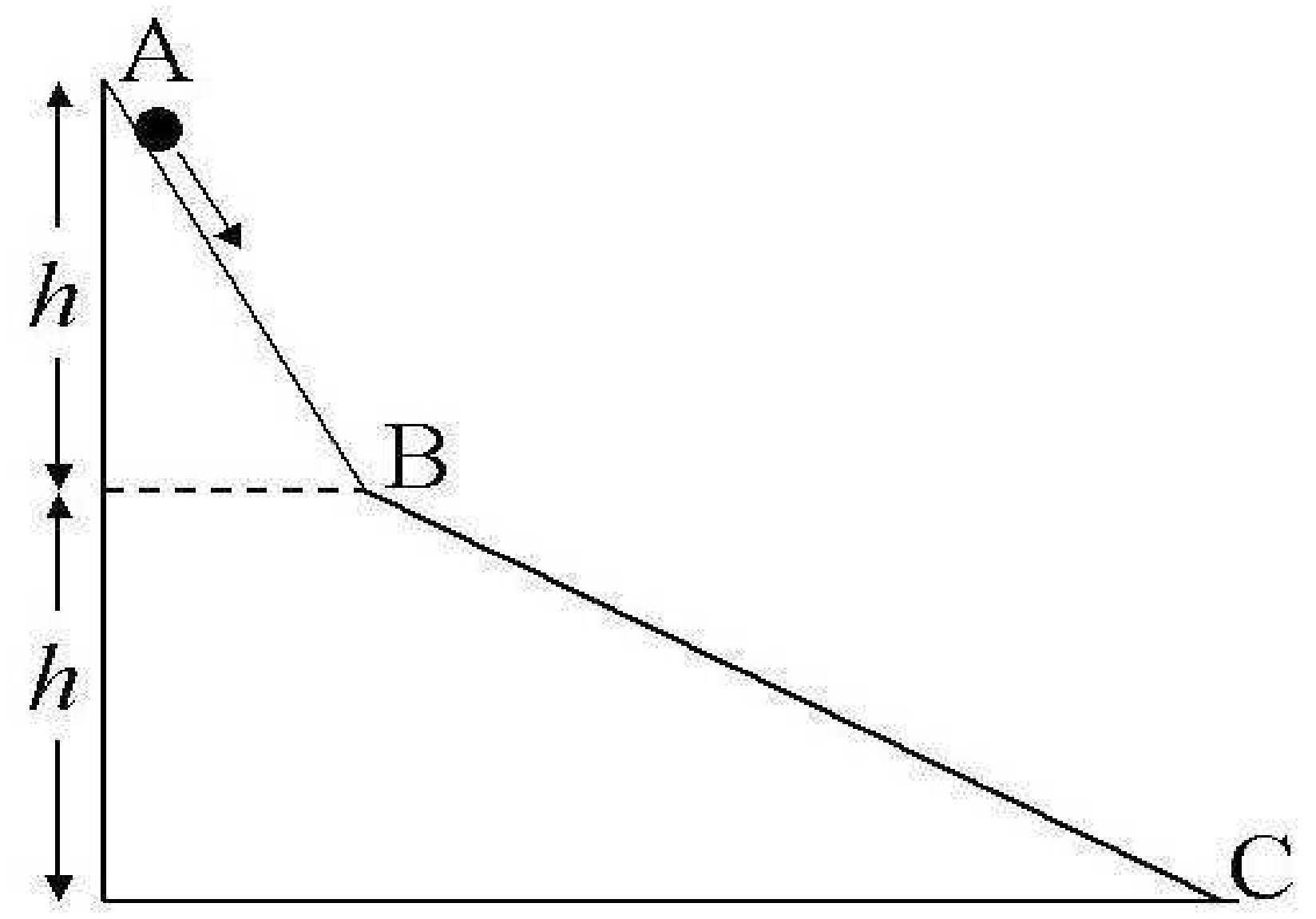




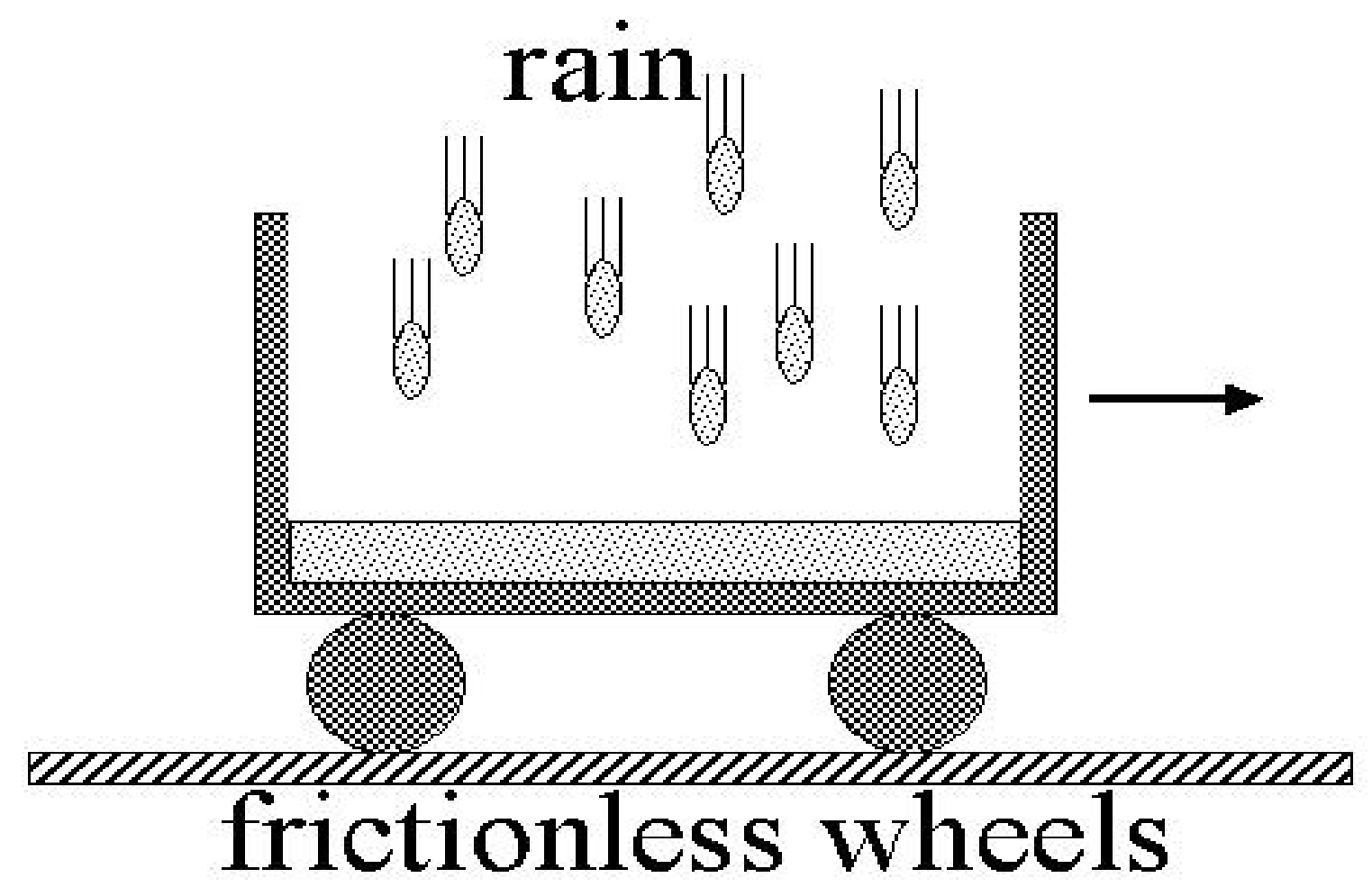

